\title{
Article
}

\section{Epidemic Location Intelligence System as Response to the COVID-19 Outbreak in Bosnia and Herzegovina}

\author{
Almir Karabegovic ${ }^{1, *}$, Mirza Ponjavic ${ }^{2}$ and Mirsada Hukic ${ }^{3}$ \\ 1 University of Sarajevo, Bosnia and Herzegovina; akarabegovic@etf.unsa.ba \\ 2 International Burch University, Sarajevo, Bosnia and Herzegovina; mirza.ponjavic@ibu.edu.ba \\ 3 Academy of Sciences and Arts of Bosnia and Herzegovina; mirsadahukic@yahoo.com \\ * Correspondence: akarabegovic@etf.unsa.ba
}

\begin{abstract}
The outbreak of COVID-19 is a public health emergency that caused disastrous results in many countries. The global aim is to stop transmission and prevent the spread of the disease. To achieve it, every country needs to scale up emergency response mechanisms, educate and actively communicate with the public, intensify infected case finding, contact tracing, monitoring, quarantine of contacts, and isolation of cases. Responding to an emergency requires efficient collaboration and a multi-skilled approach (medical, information, statistical, political, social, and other expertise), which makes it hard to define one interface for all. As actors from different perspectives and domain backgrounds need to address diverse functions, the possibility to exchange available information quickly would be desirable. Geoportal provides an entry point to access a variety of data (geospatial data, epidemiological data) and could be used for data discovery, view, download, and transformation. It helps to deal with challenges like data analysis, confirmed cases geocoding, recognition of disease dynamics, vulnerable groups identification, and capacity mapping. Predicting and modeling the spread of infection, along with application support for communication and collaboration, are the biggest challenges. In response to all these challenges, we have established the Epidemic Location Intelligence System (ELIS) using open-source software components in the cloud, as a working platform with all the required functionalities.
\end{abstract}

Keywords: geoportal; location intelligence; geospatial data; emergency response; health expert system; decision support system

\section{Introduction}

Novel coronavirus pandemic is a continuous pandemic (COVID-19) caused by severe acute respiratory syndrome coronavirus [1-3]. The epidemic is discovered in Wuhan, China, in December 2019, and announced to be a public health emergency of international concern on January 30, 2020 [1-3]. The ability to detect disease outbreaks in the early stages is a key component of efficient disease control and prevention. Increased availability of healthcare data facilitated to development of algorithms that allow more effective disease surveillance. The research focuses to develop tools for outbreak data collection, distribution, analyses, and simulation. This paper describes the system that we developed to identify and monitor disease outbreaks considering the strengths and limitations of data collection instruments, facility of location data collection, and relevant attributes for understanding disease risk. By using such information, it is quite easy for authorities to locate the highly affected area and take appropriate actions. However, GIS techniques, resources, and methods can be used for more effective investigation of epidemic, demographic, and environmental data.

Early detection of disease outbreaks is crucial for the effective control of infectious diseases. Spatial data are currently being collected but are often underused in routine surveillance of infectious diseases, especially in developing countries. As the outbreak is often characterized by the degree of spatial diffusion of cases, spatial-temporal surveillance 
algorithms are being developed in many countries. These spatial-temporal algorithms aim to facilitate the early detection of disease outbreaks that show spatial groupings, such as those associated with person-to-person disease transmission or a localized source of infection. An example is a COVID-19 exposure notification system or digital contact tracing system launched by Apple and Google for public health departments [4].

In the fight against COVID-19, geoinformation systems (GIS) have played an important role in many aspects, including the rapid aggregation of multi-source data, rapid visualization of epidemic information, spatial tracking of confirmed cases, prediction of regional transmission, spatial segmentation of the epidemic risk and prevention level, balancing and management of the supply and demand of material resources, and social-emotional guidance and panic elimination. It provided solid spatial information support for decision-making, measures formulation, and effectiveness assessment of COVID-19 prevention and control. GIS has a complete technological route for data preparation, platform construction, model construction, and map production. However, in the struggle against the widespread epidemic, the main challenge is finding strategies to adjust traditional technical methods and improve the speed and accuracy of information provided for social management.

In line with recent research [5] we also recognized the following possibilities of GIS application:

- $\quad$ capacity development and usage of GIS tools for controlling the pandemic;

- detect the onset of epidemics, identify and count cases;

- $\quad$ carry out convenient multi-scale dynamic mapping of the epidemics;

- $\quad$ spatial segmentation of the epidemic risk and prevention level;

- the tabulation and orientation of the data in terms of time, place, and person (descriptive epidemiology);

- $\quad$ implement and evaluate control and prevention measures, rapid estimation of the population flow and distribution;

- develop, test hypothesis and plan the balancing of supply and demand of medical resources;

- $\quad$ assessment of the feasibility of the supply of materials and transportation risk; and

- communicate the findings and monitor the spatial spread of social sentiment and detection.

However, its implementation implies a number of difficulties and challenges [6], some of which are discussed in this paper.

Governments, public health agencies, and academic institutions have launched different GIS implementations. The most famous example is the Johns Hopkins Covid-19 dashboard, a website assembled by the university's Center for Systems Science and Engineering, which makes the worldwide well-known interface of geoportal and GIS dashboard elements, like tally or leaderboard of confirmed cases, heat map of cumulative cases, tables, key figures, graphs. It is built using the Esri commercial technology ArcGIS, well known and used all around the world. Actually, it is easy to identify the ArcGIS dashboard, as all implementations have the same user interface. The interface is modular, each dashboard can be assembled in a different way, but most organizations the use same or very similar interface [7].

Other organizations chose to implement their own system based on open-source platforms, mostly using Leaflet or Open Layers mapping libraries [8,9]. The third route was taken by organizations that already had some e-health or business intelligence system and chose to create a public online dashboard to present data collected by those systems [10].

What is common for most of these implementations is that they are designed for a broad audience and mostly focused on data presentation of few static pieces of information such as daily testing of persons, confirmed cases, deaths, and recovered persons. By combining location-related data with other thematic data like epidemic ones, it is possible to gain critical insights, make better decisions, and optimize important processes and applications. Location Intelligence (LI) has the capacity to organize and understand 
complex phenomena, using geographic relationships, which are inherent in all information. It offers an opportunity to streamline the processes and recognize relationships to improve performance and results [11].

Bosnia and Herzegovina $(\mathrm{BiH})$ as a country with significantly underfunded public health system development has limited resources to fight the COVID-19 epidemic. The coronavirus is declared as a global pandemic and the first case is reported in BiH on March 5th, 2020 (see Figure 1). In order to respond quickly to the rapid development of the epidemic situation related to the spread of COVID-19 in BiH, the Committee for Microbiology and Related Disciplines of the Department of Medical Sciences at the Academy of Sciences and Arts of Bosnia and Herzegovina (ANUBiH) launched the project Epidemic Location Intelligence System (ELIS) to monitor the spread of the COVID-19 virus and formed a multidisciplinary team to implement it.

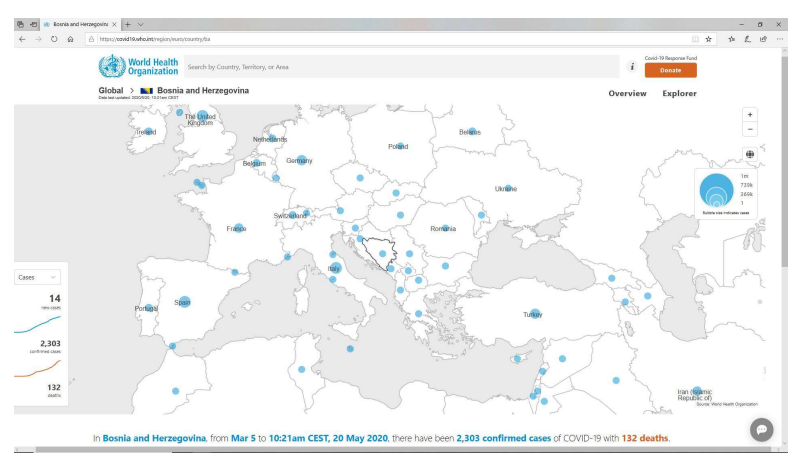

(a)

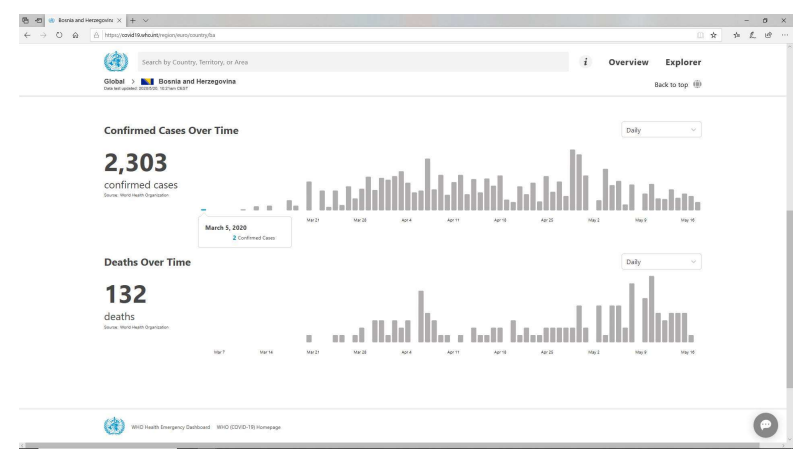

(b)

Figure 1. The outbreak of COVID-19 in Bosnia and Herzegovina. (a) Position and comparison in a number of cases in Europe; (b) Confirmed cases and deaths over time. Screenshots from https://covid19.who.int/ on May 20 th, 2020.

The system aims to provide epidemic data collection, processing and analysis, research of the COVID-19 epidemic, communication and exchange of information with public health service institutions, evaluation of the existing epidemic surveillance system and control measures taken, reporting to government institutions and the general public about the epidemic situation.

Due to the heterogeneity of health information systems and protocols defining the use of epidemic data in Bosnia and Herzegovina, a separate autonomous channel for data exchange with local public health institutes has been established to serve as a data pipeline for ELIS. Some sources of data coming from higher administrative units were publicly available on official sites of $\mathrm{BiH}$ institutions. The availability of geospatial data and the demand for better analytic insight have motivated us to move location analysis to the web environment. Most of the epidemic data have a location reference (address) which allows enhancing its analytics with location intelligence as shown in Figure 2.

In addition to the difficulties with data exchange, the paper discusses the challenges that the project team had to deal with, such as data preparation and processing for analytical and modelling purposes; geocoding of tested, confirmed, and active cases; time dimension recognition; identification vulnerable population regions and available health resources; how to model the spread prediction of COVID-19; and finally, facilitating seamless and instantaneous communication and cooperation between all actors, which was the biggest challenge in the current situation. The paper suggests an approach with location intelligence using open-source software components for ELIS development as a response to all these challenges. 


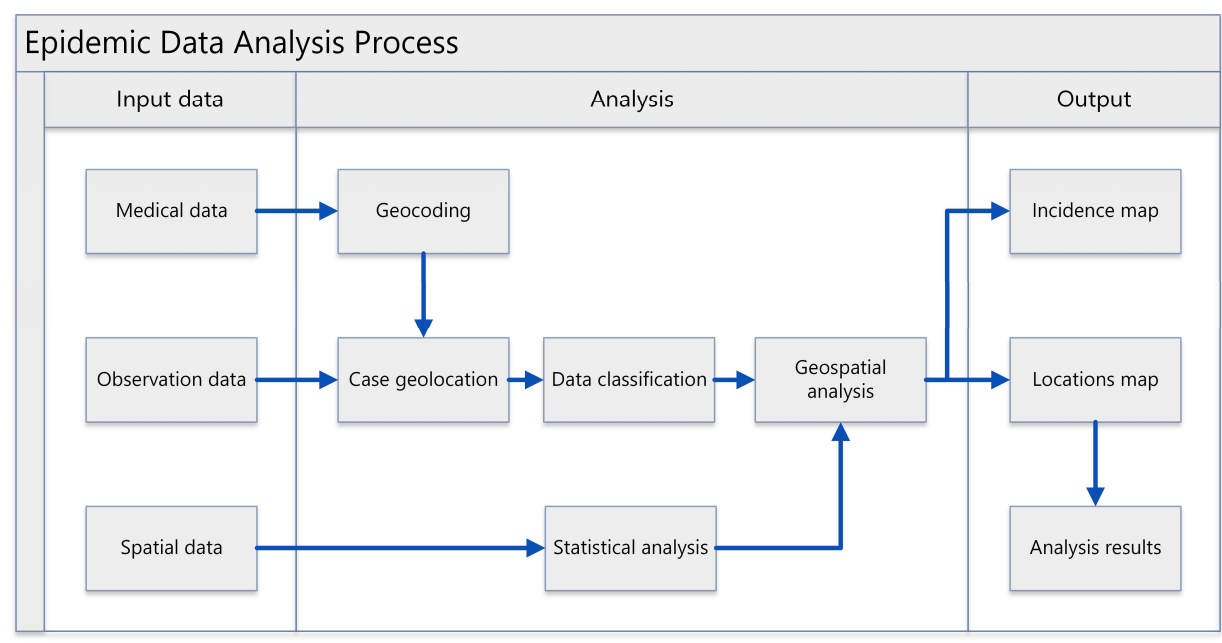

Figure 2. Epidemic data processing and spatial analysis. Input data include epidemic or observation data to be mapped and spatially analyzed. The outputs of analysis are maps and data tables, statistically analyzed for dissemination of knowledge (Adapted from [12]).

\section{Materials and Methods}

Geoinformation systems (GIS) are all about spatial analysis, and they were originally developed to store, manage, analyze, and present spatial data. Apart from the visualization, there are many important features in GIS [13]. It provides the capability to integrate location as part of an information system and can improve many types of applications through enhancing accuracy, efficiency, knowledge, and intelligence. Some GIS concepts resemble standard IS concepts, while others are not widely known in the information systems (IS) field [11,13,14]. Most GIS techniques are grouped into:

- Spatial Analysis (spatial data characteristics, map overlays, selection by attribute, selection by location, georeferencing and geocoding, classification and thematic mapping, proximity analysis, buffers, using distance during location selection, Thiessen polygons / creating proximal zones, density, and specialized modeling);

- $\quad$ Spatial Statistics; and

- $\quad$ Space-time Trend Analysis and 3D Modeling [14].

The fundamental idea of employing GIS is to explore the geographic space, distribution patterns, evolution processes, and the interactions of geographical elements to enable users to better understand the world [15].

Methodology for the establishment of the system includes four steps, data collection and centralization, system integration, data presentation, and user acceptance [16].

\subsection{Data Collection and Centralization}

Based on the idea, the authors propose a system that supports a workflow for the assessment and improvement of epidemiological surveillance, where data are taken from the electronic records of public health institutes, hospitals, and laboratories and grouped into a single epidemiological database. From this database, two data warehouses are created (spatially and epidemiologically oriented), which represent the basic data sets in ELIS that are visualized via geoportal.

Spatial data mart includes data for the whole country:

- administrative spatial units up to the level of settlements;

- basic infrastructure up to the level of streets and important facilities;

- natural characteristics (soil types, vegetation, climate, precipitation);

- demographic data up to the level of settlements (by age groups, types of households, etc.); and

- healthcare facilities, pharmacies, and other health service facilities. 
The actual availability of digital data is a result of series of projects implemented in Bosnia and Herzegovina during the previous decade, mostly to meet the operative needs of local government, and with the establishment of a centralized spatial information system for the business processes support.

Based on data research and their availability, there were chosen set of required epidemic data attributes as following for every case:

- cluster membership;

- the outcome;

- gender;

- age;

- date of examination;

- $\quad$ test site;

- hospitalization;

- addresses;

- contacts of the case; and

- symptoms.

Base datasets include official daily reported values of the cumulative number of laboratory-confirmed COVID-19 cases. Epidemic data for cantons and municipalities are taken from public health databases and available records. These data are supplemented with other transparent clinical and laboratory information needed to develop a model for predicting the spatial and temporal spread of infection.

\subsection{System Integration}

The concept and practice of openness have many components. We are most familiar with open-source software and open data, but not less important are open hardware, open standards, open education, and consequently open science. Open-source geospatial software includes a broad range of libraries, tools, applications, and platforms developed and released under different Open Source Initiative (OSI) licenses, where most used those that are part of the Open Source Geospatial Foundation (OSGeo) software ecosystem [17,18]. OpenStreetMap (OSM) is the most widely known example of a global open geospatial dataset, collaboratively maintained and expanded through a global community of contributors. OSM was inspired by restrictions on the use and availability of geospatial data all over the world.

Due to the specific situation in the country, problems with acquiring software licenses, legal procedures in procurement, the synergy between open-source geospatial software and open geospatial data together with official data could make a good working community composed of academia, civic society, the private and public sectors $[17,18]$.

There are many examples of frameworks for integrating cloud computing with GIS through architecture, workflow, and a case study in the health sector [19], promising to be valuable in solving resource distribution and diseases tracking.

Because of very sudden and unplanned inception of the ELIS project as well as the time-critical nature of the epidemic, we had to act swiftly to create a reliable ICT infrastructure. Cloud computing was the only possible solution in a such short period of time available for implementation of the system. It made handling a large amount of data and services, enabling users to scale, customizing and providing computing resources (software, storage, and development platforms), and giving access to the data and services possible, without time or location dependency. Case data can include sensitive private information about citizens like name or address, which is not suitable to record in public cloud resources.

\subsection{Spatial Data Presentation}

The geoportal is a type of web portal (web service platform) used to find and access geographic information and associated geographic services (display, editing, analysis, etc.) via the Internet in a service-oriented architecture. This kind of portals can dramatically expand the availability of location-based data to users for review, editing, and 
analysis. Modern geoportals include direct access to data (in any formats or forms), metadata, visualization tools, automated provenance linkages across users, review tools for data interpretation, and sharing results in files, papers, or directly on social networks. Many use case examples, not only government healthcare (disease outbreak tracking) but also retail (trade area analysis) or marketing (location-based marketing effectiveness) proof that it is possible to use advantages of such solutions [11].

COVID-19 spread monitoring models were selected according to the availability of epidemic data sets. The growth dynamics of the diseased are modeled using the SIR (S susceptible; I- infectious; R - recovered) model applied to two possible scenarios: pessimistic and optimistic. Also, epi-curves and reproduction number Rt are used to monitor the effectiveness of control measures to suppress the spread of infection.

These models with parameters related to the dynamics and prediction of disease spread were part of reports generated and published via the ELIS geoportal.

\subsection{User Acceptance and Usage}

End users were included in the entire process from the very beginning, preparing data and testing interface. After the first month of the development phase, they started to use the system actively, asking for additional functions, meaning that the development continued together with usage.

Users of the system were divided into three groups: data administrators, data analysts, and public users. According to the constitutional solutions, the public health system in Bosnia and Herzegovina is regulated in accordance with the administrative authorities of two entities, district and ten cantons. The users from thirteen public health institutions together with users from clinical centers and microbiological laboratories were continuously collecting, entering, and validating data. This group has varied from 20 to 30 participants using ELIS as a mostly evidence-based system for the country level.

Group of data analysts, consisting of 22 experts from these public health institutions and university clinical centers, and five faculties of medicines have used ELIS as an expert system and produced reports about spread analysis and evaluation of the epidemic situation, for different areas and time periods [20,21,22]. Geographical, demographic, and other data combined with epidemic data was processed using the selected analytical methodology, and the results were arranged in the ELIS geoportal in the form of diagrams, attribute tables, interactive maps, and animations.

The third group, public users including press workers, has used ELIS as an informative portal to understand the current situation as well as the history of spread. The number of public visitors on daily basis has been more than a hundred ones.

\section{Results and discussion}

The Epidemic Location Intelligence System (ELIS) is an expert information system for analyzing epidemic data and reporting. The functionality includes communication, analysis, and reporting, which is provided by a robust ICT infrastructure in the background. The system is based on modern design principles and service-oriented architecture, so it allows complete separation of backend and frontend as shown in Figure 3a. Due to the visual effectiveness of the geoportal interface itself, it is generally very popular, however, its functionality is far broader than the spatial data interfaces used by news portals and some government agencies to present epidemic data.

The frontend is a JavaScript web application that communicates with web services using AJAX and allows to connect different mapping libraries. The frontend is based on the ReactJS library and the Redux architecture, which is a specific implementation of the Flux architecture.

Backend is a set of web services, developed in Java and includes services for HTTPProxy, storage of unstructured resources, and security with the ability to configure authentication using internal or external service, and flexible authorization policies for access to services and resources. 


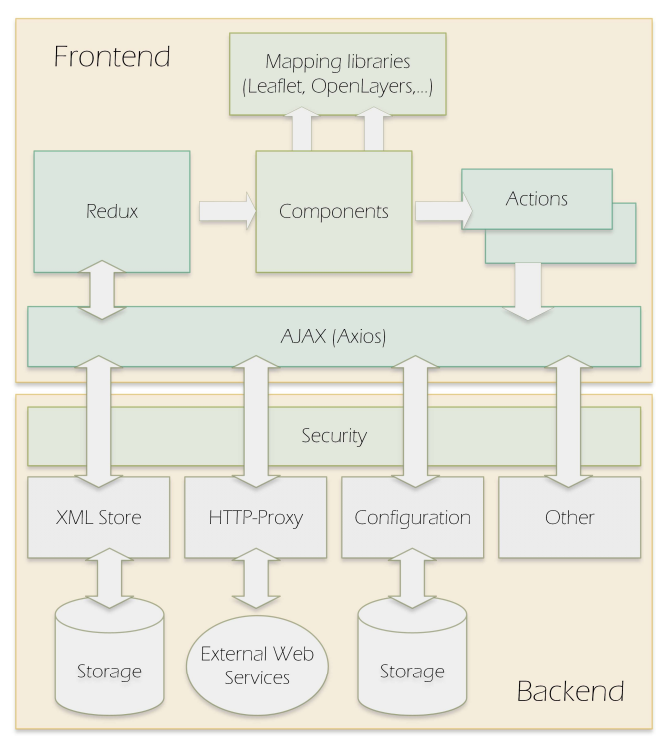

(a)

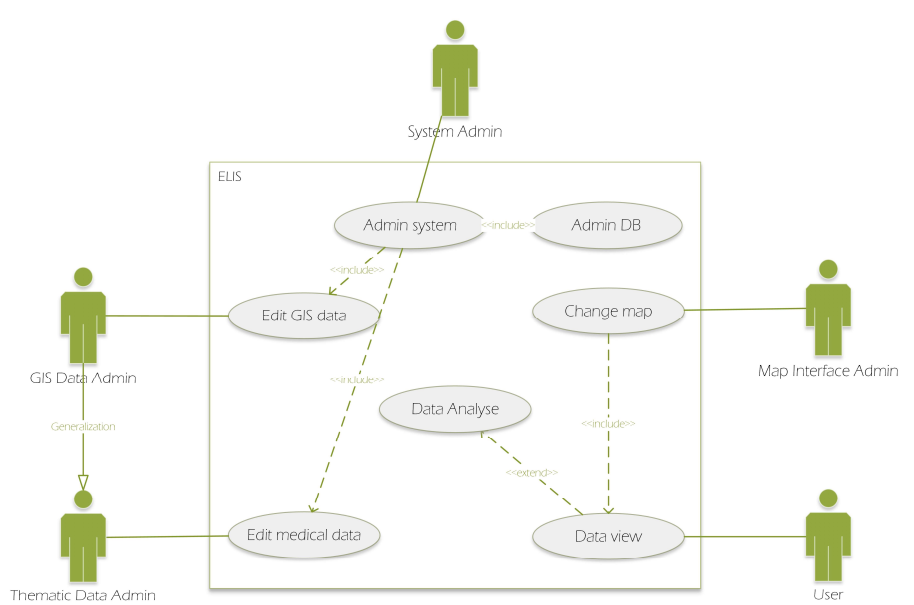

(b)

Figure 3. Epidemic Location Intelligence System (ELIS). (a) Architecture; (b) Use Case.

ELIS is based on the following tech stack:

- MapStore is an Open Source highly modular web GIS framework developed by GeoSolutions to create, manage and share securely in a simple and intuitive way maps created by mixing geospatial contents served from servers like Google Maps, OpenStreetMap, Bing, or from server adhering to OGC standards like WFS, CSW, WMS, and WMTS;

- GeoServer is an open-source server that generates maps and controls spatial data. It implements OGC standards like WMS, WFS, WCS. Its interface has tools to add, update, delete data sources and layers, as well as set styles;

- OpenLayers used for the development of front-end application as the JavaScript library which enables browsing and interacting with spatial data via web browsers; and

- $\quad$ PostgreSQL with PostGIS used as DBMS [18].

Based on this architecture and the concept of Web 2.0, the system provides a free classification of information, responsive interface with dynamic content, user participation in terms of changing data and interface, Software as a Service based on APIs. It includes standard analytical tools such as interactive maps, control panels, various diagrams, etc. The user interface, as its client segment, lets different types of users work easily, especially the non-IT-expert users. Each logged-in user is assigned the appropriate services (maps, dashboards, database schemas) [19].

The results of this structure with a hybrid cloud, and a mix of the private and public environment were:

- fast implementation - in a week we decided on the cloud provider, created needed infrastructure, and installed all applications;

- no procurement - cloud computing allows billing method pay-as-you-go, plus using an open-source model made system license-free;

- access to all services 24/7 regardless of the location and the time (user need only an internet connection);

- controlled access - system allows only authorized users to access data based on defined rules and roles; and

- data protection - sensitive data are not stored in the public cloud, they stayed in the original official store, and only aggregated on street or settlement levels for analytical purposes. 
In general, the portal, as a web user interface, is an access point to many resources. It provides an environment for users to access the data. Portal provides links to other websites and includes various security measures, including access rights to the portal or parts of it. It can be personalized to fit the needs of the user in terms of functionality and appearance. Its development aims to promote the interoperability of data and services, which is achieved through the acceptance and implementation of the standards. Interoperability is largely achieved through standardized geoportal architecture. Users are separated into two main categories: GIS data administrators and Case data ones.

Along with choosing required components to develop and deploy the web system, there are considered technical standards that allow interaction between these components [23]. Open Geospatial Consortium (OGC) defined standards like Geography Markup Language (GML), which is based on Extensible Markup Language (XML), data identification such as the Catalog Service for the Web (CSW), data provision services such as the Web Mapping/Feature/Coverage Service (WMS/WFS/WCS), and spatial processing such as the Web Processing Service (WPS) specification, based on the Hypertext Transfer Protocol (HTTP). To integrate geospatial data and services with web-based distributed applications, the OGC specified Web Service implementation standards (OWS) interface.

The methodological approach and carefully selected tech stack helped to provide answers to the challenges faced by the Project team:

\subsection{Data analysis and modeling}

Data analysis and modeling involve the process of extracting, transforming, and loading data into a database. These data include three types of source:

1. official (from the Institute of Public Health and competent institutions),

2. reliable (from private sources and industry), and

3. public (from the portal of news agencies and social networks).

Given the diversity of data sources, formats, structures, and the frequency and level of data acquisition, appropriate mechanisms are provided for the transformation of raw data into appropriate models.

Data modelling ensured that all available data was placed within a logical model implemented in the DBMS user environment. The proposed graphical and alphanumeric data model is consistent with the database model and the metadata catalog based on Core Metadata for Geographic Datasets - ISO 19115: 2003 (E) standard. The geometric data model is based on available geometric types of GML 3.1.1. standardized geometric data model (spatial topology), as well as associated complex types that enable the maintenance of GIS data using a time-domain (time topology) according to ISO 19107: 2003 (geographic information - spatial schema) and ISO 19108: 2002 (geographic information - temporal schema) standards [18].

Generally, spatial data go through three phases. The first phase is their acquisition, the second one is storing in databases (to be accessible for analysis, search, querying, or further processing), and the third phase is their presentation (maps, tables, diagrams, text). Each of these phases has distinctive requirements on how to store and keep data, so the modelling of the data considers different models [21]:

- acquisition-oriented data model - receives the data in sequences and according to the structure appropriate to the way and method of their collection;

- $\quad$ query-oriented data model - where the data are stored in the database so that they are accessible for query execution; and

- $\quad$ output-oriented data model - supports the display of results (outputs) of query operations in the form of tables, maps, reports, and other display formats.

\subsection{Geocoding of cases}

According to the definition, geocoding is the process of translating street addresses into geographic latitude and longitude coordinates for accuracy. Geocoding of epidemic cases enables mapping of tested, confirmed, and active cases, and outcomes in terms of 
death or recovery. Different address models, official, private, and open (subject to availability) are used to identify where COVID-19 infections have occurred.

ELIS use OpenStreetMap (OSM), a publicly available geographic database from around the world. Using the addresses contained in the OSM, the system geolocated the cases and put them on a map. An important issue was data confidentiality. In order to respect anonymity, health data are usually aggregated at the level of the administrative unit, which, in turn, leads to a loss of knowledge about the local spatial distribution of data. Authors applied this method to epidemic data creating points as cluster representatives for settlements.

\subsection{Time dimension recognition and disease dynamics}

Maps with a time dimension included can visualize the spread of infection over time (Figure 4) and recognize clustering phenomena. The capability of a geoportal for visualizing large amounts of information interactively is often sensed as the only important function of it. This ability to create multiple perspectives enhances a viewer's perceptive to understand studied phenomena.

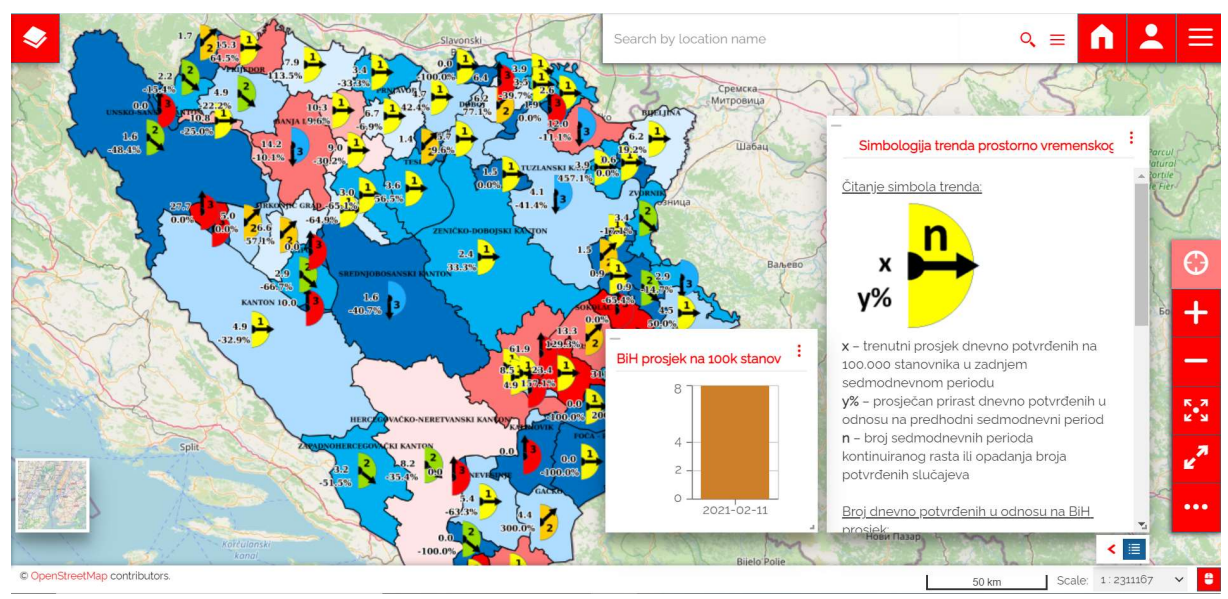

Figure 4. COVID-19 infection spread presented simultaneously by three indicators: speed, intensity, and tendency. The figure shows a diagram of the weekly average infected per 100.000 citizens. Symbols present trend (increase/decrease) for previous three weeks, and rate of weekly percentage infected comparing to the previous period in local communities.

The user interface is required to be intuitive and simple to learn, as shown in Figure 5. Generally, interactive map application allows users to explore and recognize spatialtemporal patterns.

Analogous to its positional and attribute components, the quality of spatial data can also be evaluated in terms of their temporal accuracy. This includes not only the accuracy and precision of the measurement time (for example, the date of examination) but also the temporal consistency of different data sets (which may be in conflict). As the geometric and attribute components of spatial data can be changed together or independently, their temporal validity must also be considered. Validity of the data set implies the actuality of the data in terms of the time difference between the currently available data and the actual state of the phenomenon they represent. The frequency of updating data has a direct impact on validity [21]. 


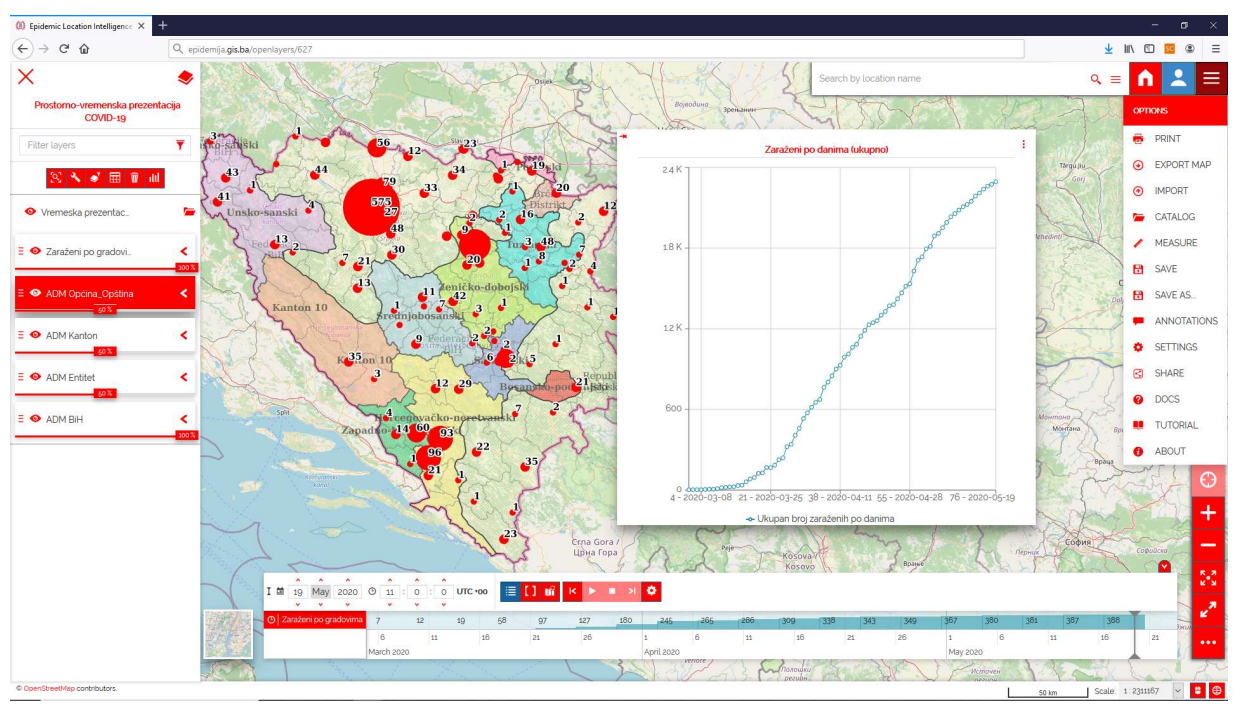

Figure 5. Elements of the system interface in Epidemic Location Intelligence System. The diagram shows the cumulative total number of infected.

\subsection{Recognizing vulnerable population regions}

The degree and severity of the disease can vary when the population from certain demographic groups is affected, and therefore age groups and other characteristics related to the population susceptibility factor are mapped, which can help control risk groups and regions. The most important factors considered for vulnerable regions are related to:

- presence of virus,

- chronic disease and age, and

- personal protection.

\subsection{Capacity mapping}

Services, medical resources, equipment, and other resources related to health care facility locations are mapped in order to more easily assess the readiness of the public health system to respond effectively to the current epidemic situation. Important quantitative information mapped are:

- hospital locations;

- total number of beds in hospitals;

- available beds;

- total number of ventilators in hospitals; and

- available ventilators in hospitals.

\subsection{Modelling and prediction}

Models of spreading the infection are created with the prediction of the occurrence and warn of possible risks. The system displays all reports in one place with a clear indication of the geographical area and time interval they covered. They make comparing the effects of control measures easier (Figure 6.). Based on these assumptions, the project team has developed themes for the current state based on existing cases, and predicted conditions based on statistics.

Current state themes are:

1. dispersion of cases by addresses with time presentation;

2. cumulative disease rate per municipalities (with a percentage share in total and the ratio "per thousand inhabitants");

3. overview of confirmed cases per settlements by annotation and choropleth map;

4. list of all tested per settlements by annotation of number of tested and choropleth maps and ratio tested in the settlement "per thousand inhabitants" by annotation; and 
5. cumulative presentation of the ratio of cases by sex and age for the passive and active population with diagrams showing the statistics of diseased.

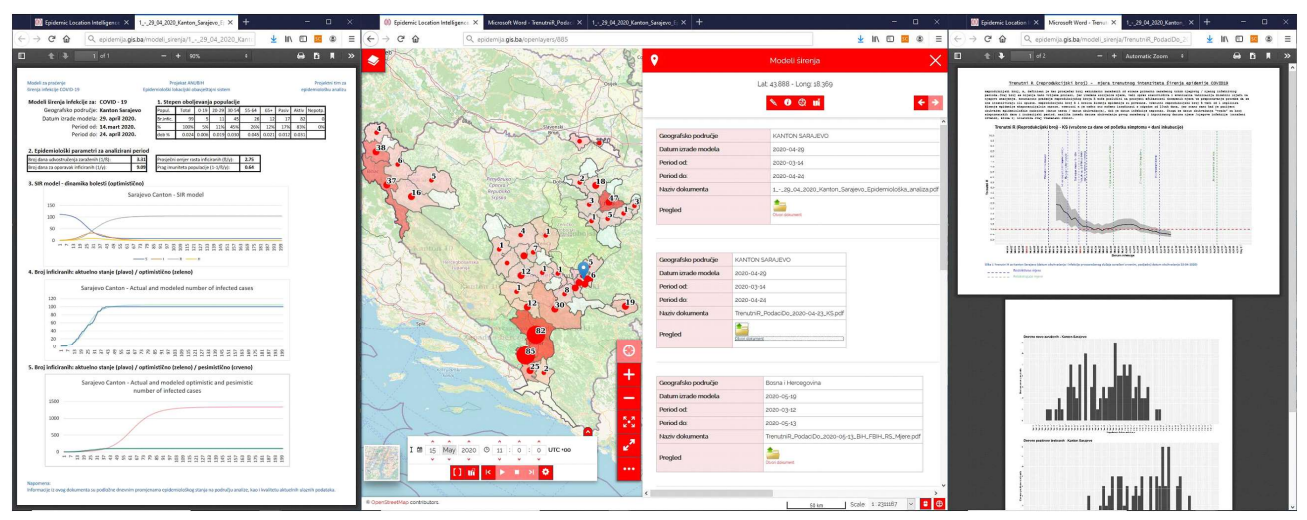

Figure 6. Modeling and prediction of spreading the infection in Epidemic Location Intelligence System. The figure shows current reports with epidemic curves and instantaneous weekly reproductive number (Rt).

Predicted conditions themes are:

- $\quad$ expected total number of cases with needs for health care in relation to the entire population;

- $\quad$ total cumulative number of hospitalized (with mild, moderate, and severe symptoms) and isolated (supervised) and number of cases of respirator use;

- $\quad$ expected increase of cases for the next 7 days (optimistic and pessimistic);

- $\quad$ epidemic curves (Epi-curves) [20,21]; and

- $\quad$ instantaneous (weekly) reproductive number (Rt) to control the effects of the applied measures [20,21].

\subsection{Communication and cooperation}

Interactive maps and control panels are used between participants to quickly communicate about the current situation during the monitoring and to be informed about the expected development of the situation. Interactive dashboards, like one shown in Figure 7 , are a particularly useful tool because they display key indicators and provide summary critical information.

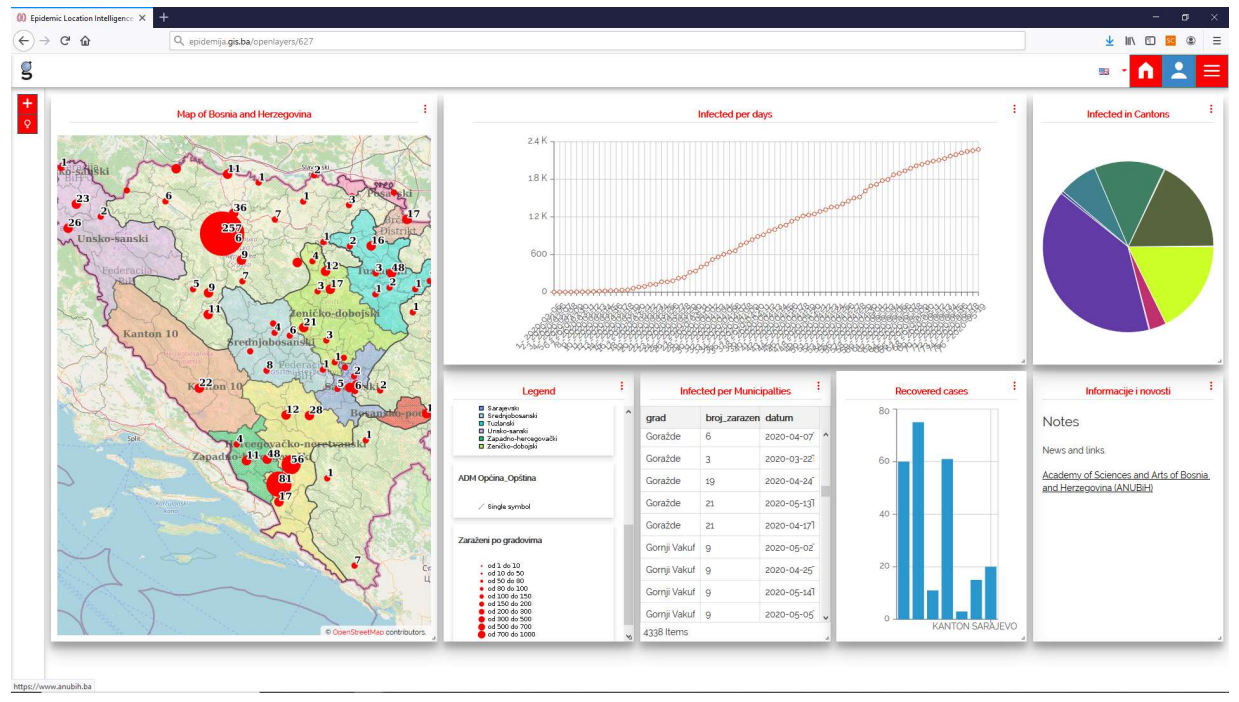

Figure 7. The dashboard displays key indicators and provides summary critical information. 


\section{Conclusions}

The limitations of the public health system are reflected in the partial approach to recording and reporting data in Bosnia and Herzegovina and in a number of heterogeneous data sources, which are overcome by ELIS implementation.

In response to the challenges which every country faces with the outbreak of COVID19 as a public health emergency, we established an epidemic location intelligence system (ELIS) using open-source components. This approach enables data modeling with a time dimension, vulnerable population regions, and capacity mapping, prediction, stakeholders communication, and cooperation.

Geoportal, with such structured data, allow access to information from different sources of local, national, and global level in an interoperable manner and for a wider range of users. This access to relevant, harmonized and quality information is critical for decision-making in a public health emergency. Additionally, this interactive component with a data warehouse in the background represents a knowledge-based system intended to help decision-makers to compile useful information from a combination of raw data, documents, and personal knowledge. This decision-making support system gathers and presents information like inventories of information assets, comparative statistic and demography figures between time points, and historic and predicted key indicators and natural characteristics based on statistic assumptions. We recognized the best features of geoportals and its services: portal services that provide access to geospatial data and manage portals; presentation services used to process geospatial data and prepare for presentation to the user; catalog services for locating geoservices providing information; and data services for accessing and downloading geospatial content. In general, portals do not store data, but datasets remain with their owners who retain control over them. Users of the services can view data and perform certain operations on them but cannot edit or save them on their system without permission and official download. This is useful for the general public, but it is restrictive for health experts, who need more freedom in data manipulation. The fact required the design of a complete spatial decision support system, with geoportal as interface. This system is developed with interfaces that implement geospatial processes to meet domain-specific needs which vary from one research area to another. In this way, an expert involved in its field can use GIS functionality, geoinformation, and related geoprocesses in one web environment.

The system development concept was based on the principles of openness. Without the need to deal with software licenses, the complete system was ready in the week and installed in a cloud computing environment. Thanks to using the standards, acquiring data from public sources started immediately, and was fast followed with data from the public site of health authorities. Using open geospatial software, data and standards made the development of the system direct and fast.

All the above created an atmosphere of trust allowing the next step to obtain data directly from the official sources. This was followed by epidemic data analysis including the application of appropriate data processing algorithms and methods for presenting the outcomes. We reported the results by using advanced features like dashboards, maps, charts, epi-curves, SIR, and Rt diagrams.

And, among its other purposes, ELIS will serve in perspective as a research base of interest for the scientific community in the country and the region.

Supplementary Materials: Public version of ELIS is available online at http://epidemija.gis.ba/.

Author Contributions: Conceptualization and Writing, A.K., M.P. All authors have read and agreed to the published version of the manuscript.

Funding: This research was funded by the Academy of Sciences and Arts of Bosnia and Herzegovina (ANUBiH) and the Ministry for Science, Higher Education, and Youth, Canton Sarajevo.

Data Availability Statement: Public version of ELIS is available online at http://epidemija.gis.ba/. 


\begin{abstract}
Acknowledgments: The authors belong to the Project team formed by theCentre for Control Disease and Geo-Health Studies at the Academy of Sciences and Arts of Bosnia and Herzegovina (ANUBiH) and would like to thank the rest of its members for their support, as well as Public Health Institutions in Bosnia and Herzegovina.
\end{abstract}

Conflicts of Interest: The authors declare no conflict of interest.

\title{
References
}

1. World Health Organization. (2020). Considerations for quarantine of individuals in the context of containment for coronavirus disease (COVID-19): interim guidance, 19 March 2020.

2. World Health Organization. (2020). Critical preparedness, readiness and response actions for COVID-19: interim guidance, 22 March 2020.

3. World Health Organization. (2020). COVID-19 Strategic Preparedness and Response Plan: Operational Planning Guidelines to Support Country Preparedness and Response, 12 February 2020.

4. Coronavirus (COVID-19) - Apple and CDC. Available online: https://www.apple.com/covid19/ (accessed on 21 May 2020).

5. Sarwar, S.; Waheed, R.; Sarwar, S.; Khan, A. COVID-19 Challenges to Pakistan: Is GIS Analysis Useful to Draw Solutions? Science of The Total Environment 2020, 730, 139089.

6. Zhou, C.; Su, F.; Pei, T.; Zhang, A.; Du, Y.; Luo, B.; Cao, Z.; Wang, J.; Yuan, W.; Zhu, Y.; Song, C.; Chen, J.; Xu, J.; Li, F.; Ma, T.; Jiang, L.; Yan, F.; Yi, J.; Hu, Y.; Liao, Y.; Xiao, H. COVID-19: Challenges to GIS with Big Data. Geography and Sustainability 2020, 1 (1), 77-87.

7. Johns Hopkins Coronavirus Resource Center. Available online: https://coronavirus.jhu.edu/ (accessed on 21 May 2020).

8. Alamo, T.; Reina, D.G.; Mammarella, M.; Abella, A. Covid-19: Open-Data Resources for Monitoring, Modeling, and Forecasting the Epidemic. Electronics 2020, 9, 827. https://doi.org/10.3390/electronics9050827

9. Konicek, J.; Netek, R.; Burian, T.; Novakova, T.; Kaplan, J. Non-Spatial Data towards Spatially Located News about COVID-19: A Semi-Automated Aggregator of Pandemic Data from (Social) Media within the Olomouc Region, Czechia. Data $2020,5,76$. https://doi.org/10.3390/data5030076

10. Sechi, G. M., Migliori, M., Dassi, G., Pagliosa, A., Bonora, R., Oradini-Alacreu, A., Odone, A., Signorelli, C., Zoli, A., \& Response Team, A. C. Business Intelligence applied to Emergency Medical Services in the Lombardy region during SARS-CoV-2 epidemic. Acta bio-medica 2020, 91(2), 39-44. https://doi.org/10.23750/abm.v91i2.9557

11. Karabegovic, A.; Ponjavic, M. Geoportal as Interface for Data Warehouse and Business Intelligence Information System. Advances in Intelligent Systems and Computing Advances in Business ICT 2014, 27-40.

12. Herbreteau, V.; Révillion, C.; Trimaille, E. GeoHealth and QuickOSM, Two QGIS Plugins for Health Applications. QGIS and Generic Tools 2018, 257-286.

13. Kainz, W. Cartography and the Others - Aspects of a Complicated Relationship. Geo-spatial Information Science 2020, 23 (1), 5260.

14. Farkas, D.; Hilton, B.; Pick, J.; Ramakrishna, H.; Sarkar, A.; Shin, N. A Tutorial on Geographic Information Systems: A Ten-Year Update. Communications of the Association for Information Systems 2016, 38, 190-234.

15. Lü, G.; Batty, M.; Strobl, J.; Lin, H.; Zhu, A.-X.; Chen, M. Reflections and Speculations on the Progress in Geographic Information Systems (GIS): a Geographic Perspective. International Journal of Geographical Information Science 2018, 33 (2), $346-367$.

16. Karabegovic, A.; Ponjavic, M.; Ferhatbegovic, E.; Karabegovic, E. Spatial Data and Processes Integration in Local Governance of Bosnia and Herzegovina. 2018 41st International Convention on Information and Communication Technology, Electronics and Microelectronics (MIPRO) 2018.

17. Coetzee, S.; Ivánová, I.; Mitasova, H.; Brovelli, M. Open Geospatial Software and Data: A Review of the Current State and A Perspective into the Future. ISPRS International Journal of Geo-Information 2020, 9 (2), 90.

18. Ponjavic, M.; Karabegovic, A. Location Intelligence Systems and Data Integration for Airport Capacities Planning. Computers 2019, $8(1), 13$.

19. Helmi, A. M.; Farhan, M. S.; Nasr, M. M. A Framework for Integrating Geospatial Information Systems and Hybrid Cloud Computing. Computers \& Electrical Engineering 2018, 67, 145-158.

20. Hukic, M.; Ponjavic, M.; Tahirovic, E.; Karabegovic, A.; Ferhatbegovic, E.; Travar, M.; Serdarevic, F. SARS-CoV-2 Virus Outbreak and the Emergency Public Health Measures in Bosnia and Herzegovina: January - July, 2020. Bosn J of Basic Med Sci 2021, 21, 111-116.

21. Ponjavic, M., Karabegovic, A., Ferhatbegovic, E., Tahirovic, E., Uzunovic, S., Travar, M., Pilav, A., Mulic, M., Karakas, S., Avdic, N., Mulabdic, Z., Pavic, G., Bico, M., Vasilj, I., Mamic, D., \& Hukic, M., Spatio-temporal data visualization for monitoring of control measures in the prevention of the spread of COVID-19 in Bosnia and Herzegovina, Medicinski glasnik, 2020, 17(2). https://doi.org/10.17392/1215-20

22. Hukic, M.; Ponjavic, M.; Tahirovic, E.; Karabegovic, A.; Ferhatbegovic, E.; Travar, M.; Serdarevic, F. SARS-CoV-2 Virus Outbreak and the Emergency Public Health Measures in Bosnia and Herzegovina: January - July, 2020. Bosn J of Basic Med Sci 2021, 21, 111-116.

23. Evangelidis, K.; Ntouros, K.; Makridis, S.; Papatheodorou, C. Geospatial Services in the Cloud. Computers E Geosciences 2014, 63, 116-122. 